\title{
CMT2Q-causing mutation in the Dhtkd1 gene lead to sensory defects, mitochondrial accumulation and altered metabolism in a knock-in mouse model
}

Chun-jie Luan ${ }^{1 \dagger}$, Wenting Guo ${ }^{1,2,3+}{ }^{\mathbb{D}}$, Lei Chen ${ }^{1}$, Xi-wei Wei ${ }^{1}$, Yimin He ${ }^{1}$, Yan Chen ${ }^{1}$, Su-ying Dang ${ }^{1}$, Robert Prior ${ }^{2,3}$, Xihua Li ${ }^{4}$, Ying Kuang ${ }^{5}$, Zhu-gang Wang ${ }^{1,5,6}$, Ludo Van Den Bosch ${ }^{2,3}$ and Ming-min Gu ${ }^{1 *}$

\begin{abstract}
Charcot-Marie-Tooth disease (CMT) is a group of inherited neurological disorders of the peripheral nervous system. CMT is subdivided into two main types: a demyelinating form, known as CMT1, and an axonal form, known as CMT2. Nearly 30 genes have been identified as a cause of CMT2. One of these is the 'dehydrogenase E1 and transketolase domain containing 1' (DHTKD1) gene. We previously demonstrated that a nonsense mutation [c.1455 $T>G\left(p . Y 485^{*}\right)$ ] in exon 8 of DHTKD1 is one of the disease-causing mutations in CMT2Q (MIM 615025). The aim of the current study was to investigate whether human disease-causing mutations in the Dhtkd1 gene cause CMT2Q phenotypes in a mouse model in order to investigate the physiological function and pathogenic mechanisms associated with mutations in the Dhtkd1 gene in vivo. Therefore, we generated a knock-in mouse model with the Dhtkd $1^{Y 486^{*}}$ point mutation. We observed that the Dhtkd1 expression level in sciatic nerve of knock-in mice was significantly lower than in wild-type mice. Moreover, a histopathological phenotype was observed, reminiscent of a peripheral neuropathy, including reduced large axon diameter and abnormal myelination in peripheral nerves. The knock-in mice also displayed clear sensory defects, while no abnormalities in the motor performance were observed. In addition, accumulation of mitochondria and an elevated energy metabolic state was observed in the knock-in mice. Taken together, our study indicates that the Dhtkd $1^{Y 486^{*}}$ knock-in mice partially recapitulate the clinical phenotypes of CMT2Q patients and we hypothesize that there might be a compensatory effect from the elevated metabolic state in the knock-in mice that enables them to maintain their normal locomotor function.
\end{abstract}

\section{Introduction}

The name Charcot-Marie-Tooth disease (CMT) originates from the French neurologists Jean Martin Charcot and Pierre Marie, as well as the English neurologist Howard Tooth, who first described the disease in 1886 [1]. The clinical name for CMT is 'hereditary motor and

\footnotetext{
* Correspondence: gumm@sjtu.edu.cn

${ }^{+}$Chun-jie Luan and Wenting Guo contributed equally to this work. 'Department of Medical Genetics, E-Institutes of Shanghai Universities, Shanghai Jiao Tong University School of Medicine (SJTUSM), 280 South Chongqing Road, Shanghai 200025, People's Republic of China Full list of author information is available at the end of the article
}

sensory neuropathy' and it is one of the most common inherited disorders of the peripheral nervous system with a prevalence of 1 in 2500 individuals [2]. Clinically, CMT is characterized by progressive muscular and sensory defects starting at the distal extremities with chronic atrophy and weakness [3]. Based on the upper limb motor nerve conduction velocities (MNCVs) (median or ulnar nerve), CMT is classified into two main forms: a demyelinating (CMT1) and an axonal form (CMT2). Furthermore, accompanying with a family history of the neuropathy, CMT1 is defined when 
MNCVs are $\leq 35 \mathrm{~m} / \mathrm{s}$ and CMT2 is defined when MNCVs are $\geq 45 \mathrm{~m} / \mathrm{s}$. Intermediate forms of CMT also exist, which are characterized by MNCVs between 35 and $45 \mathrm{~m} / \mathrm{s}$. Genetically, CMT is a heterogeneous group of diseases with more than 80 disease-associated genes identified to date [4]. At least 29 unique genes have been associated with CMT2, which represents 25 to $30 \%$ of all CMT cases (http://www.molgen.ua.ac.be/CMTMutations/default.cfm).

Several CMT2-causing genes are mitochondria- specific genes, and/or lead to defects in mitochondrial function, axonal transport, and/or in the axonal cytoskeleton $[2,5,6]$. As a consequence, mitochondrial dysfunction has been suggested as a critical pathological component of the disease mechanisms of CMT2. Moreover, these deficits in mitochondrial function and transport could dramatically affect the peripheral nerves by depriving the distal axon of an important energy source [6-8].

In 2012, we identified a new gene responsible for an autosomal dominant axonal form of CMT in a fivegeneration family with CMT2 which had been classified as CMT2Q [MIM 615025] [9]. We discovered a nonsense mutation, c.1455 T > G (p.Y485*), in exon 8 of DHTKD1. The DHTKD1 gene encodes a 'dehydrogenase E1 and transketolase domain-containing 1' protein that is hypothesized to interact with proteins involved in mitochondrial energy production [9-11]. We observed that DHTKD1 mRNA expression levels in peripheral blood of CMT2Q patients decreased to half compared with unaffected individuals [9]. Similarly, in vitro studies showed that mutant mRNA and truncated DHTKD1 were significantly decreased by nonsense-mediated mRNA decay (NMD) [9] In addition, DHTKD1 silencing was found to lead to impaired energy production. These data demonstrated that CMT2Q could be caused by the nonsense mutation in DHTKD1 [9]. Subsequently, we reported a strong correlation of DHTKD1 expression levels with ATP production, revealing that DHTKD1 plays a critical role in energy production through mitochondrial biogenesis and function maintenance $[9,12]$. However, the pathogenic mechanisms underlying axonal CMT2Q remain elusive.

In an attempt to recapitulate the phenotype of CMT2Q, as well as to investigate the $D h t k d 1^{Y 486^{*}}$ point mutation in vivo, we generated a $D h t k d 1^{Y 486^{*}}$ knock-in mouse model. We observed that the knock-in mice showed some features comparable to human CMT2Q. These include lower Dhtkd1 level, reduced axon diameter, abnormal myelination, and sensory defects. While these mice did not show any abnormalities in their locomotor performance, we found accumulation of mitochondria and elevated energy metabolism in $D h t k d 1^{Y 486^{*} / Y 486^{*}}$ mice. This is contradictory to the low energy state in our previous in vitro study. Therefore, we propose that a compensatory mechanism exists in the peripheral nervous system of the $D h t k d 1^{Y 486^{*}}$ knockin mice through an elevation of their metabolic state.

\section{Results}

Development of a Dhtkd $1^{Y 486^{*}}$ knock-in mouse model that mimics NMD observed in CMT2Q patients

To generate the $D h t k d 1^{Y 486^{*}}$ knock-in mice, we constructed a targeting vector using the Red/ET cloning method and used homologous recombination in the

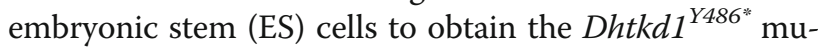
tation in mice [13]. The translated region of the mouse Dhtkd 1 gene includes 17 exons spanning approximately 2766 bp on mouse chromosome 2 . The genomic clones were isolated from a $129 / \mathrm{SvJ}$ mouse bacterial artificial chromosome (BAC) genomic library that contained genomic fragments encompassing the whole mouse $D h t k d 1$ gene. The targeting vector for the $D h t k d 1^{Y 486^{*}}$ mutation was constructed using a plasmid encompassing exons 7-10 of the Dhtkd1 gene. Two partially complimentary oligonucleotides were used to introduce the Y486* point mutation into the targeting vector. Part of the intron between exon 8 and exon 9 was replaced by PGK-Neo (Fig. 1a). Nine ES cell clones were identified as being positive by polymerase chain reaction (PCR) using primers $\mathrm{P} 1$ and P2 directed to the $5^{\prime}$ arm and primers $\mathrm{P} 3$ and $\mathrm{P} 4$ to the $3^{\prime}$ arm (Fig. 1b). Two independent ES cell clones (8A and $2 \mathrm{H}$ ) that contain heterozygous $D h t k d 1^{Y 486^{*}}$ mutation were confirmed by Sanger sequencing (Fig. 1c). Interestingly, we observed that the Dhtkd1 mRNA reduced by over $50 \%$ in heterozygous ES cell clones compared to wild-type (wt) ES cells (Fig. 1d). Following this, the two confirmed ES cell clones were injected into pseudo-pregnant females to generate chimeras. The germline transmission of the $D h t k d 1^{Y 486^{*}}$ mutation was confirmed by PCR analysis on genomic DNA. Subsequently, male chimeras were crossed with C57BL/6 J females to establish strains with a mixed genetic background (C57/129) which were heterozygous for the mutant allele. $D h t k d 1^{Y 486^{\prime \prime} /+}$ mice were bred to generate $D h t k d 1^{Y 486^{*} / Y 486^{*}}, D h t k d 1^{Y 486 \% /+}$ and wt $\left(D h t k d 1^{+/+}\right)$ littermates. Genotypes were determined by PCR using specific primers targeting either the mutant or wt allele (P7 and P2 to the Dhtkd1 ${ }^{\mathrm{Y} 486^{\circ}}$ allele and P7 and P8 to the wt allele) (Fig. 1e). The presence of the Y486* point mutation was further validated by Sanger sequencing (Fig. 1f). The cumulative genotype ratios (Dhtkd1 ${ }^{\text {Y486"/Y486", }}$ $D h t k d 1^{Y 486^{*} /+}$ and wt) for all mice are in line with the Mendelian ratios (1:2:1) (Sup. Table 1). Both $D h t k d 1^{Y 486^{*} / Y 486^{*}}$ and $D h t k d 1^{Y 486^{* /+}}$ mice had a normal life span with normal body weight similar to wt mice (Sup. Figure $1 \mathrm{~A}-\mathrm{B})$.

In addition, we also checked the expression profiles of Dhtkd1 in different tissues from wt C57/129 mice. The real-time PCR assay showed that Dhtkd1 is expressed widely in various organs and it is especially high in sciatic nerve, which is the largest nerve in the peripheral nervous system (Sup. Figure 2). In line with our previous 


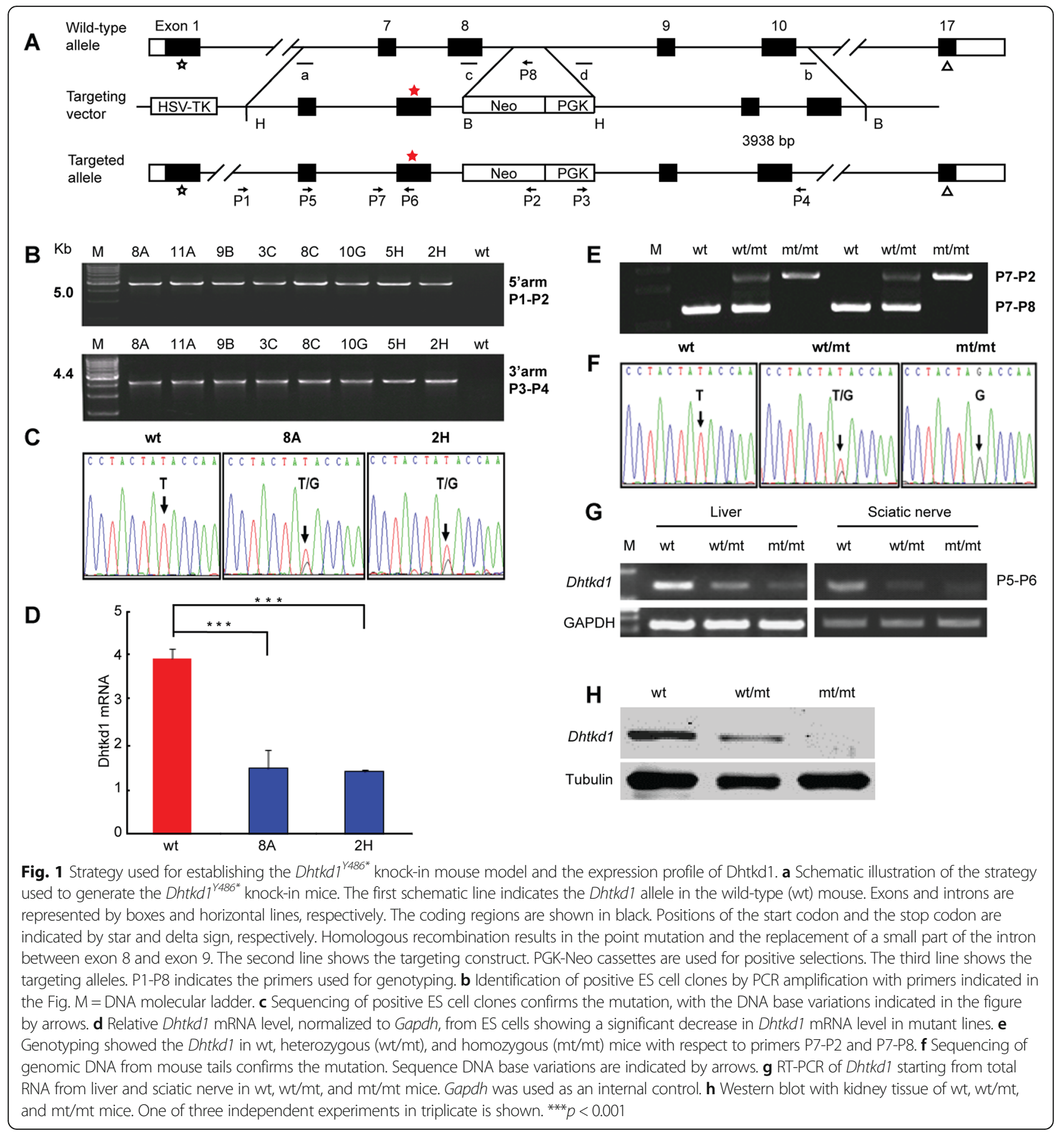

in vitro study, we observed a gradual decrease of the Dhtkd1 expression level in wt, Dhtkd1 $1^{Y 486 * /+}$ and $D h t k d 1^{Y 486^{*} / Y 486^{*}}$ in Dhtkd1 abundant-expressing tissues including liver and sciatic nerve (Fig. 1g-h) [14]. Compared with wt mice, $D h t k d 1^{Y 486 " /+}$ mice showed more than $50 \%$ reduction of $D h t k d 1$ mRNA and protein expression, while $D h t k d 1^{Y 486^{*} / Y 486^{*}}$ mice had very low Dhtkd1 mRNA and protein expression (Fig. 1g-h). Additionally, Dhtkd1 expression in the heterozygous ES cells was over
$50 \%$ reduced (Fig. 1d). As a consequence, the Dhtkd1 $1^{\text {Y486" }}$ knock-in mouse model mimicks the NMD phenomenon previously reported in patients that displayed an approximately 50\% reduction in DHTKD1 [9].

\section{Dhtkd ${ }^{\text {Y486* }}$ mice exhibit an axonopathy, but don't show locomotor defects}

To identify whether Dhtkd1 mutations lead to neuropathological hallmarks reminiscent to CMT, the distal 
sciatic nerve from $D h t k d 1^{Y 486^{*} / Y 486^{*}}, D h t k d 1^{Y 486^{* /+}}$ and their wt littermates were examined using transmission electron microscopy (TEM) (Fig. 2a-f). Prominent myelin infoldings and axonal degeneration were observed in the Dhtkd1 $1^{Y 486^{\prime \prime} / Y 486^{*}}$, Dhtkd1 $1^{Y 486^{\prime \prime} /+}$ mice (Fig. 2b and c). A higher degree of axonal degeneration, myelin infoldings, and 'double' myelination was observed in the homozygous $D h t k d 1^{Y 486^{\prime \prime} / Y 486^{*}}$ mice (Fig. 2d-f). Interestingly, TEM revealed a reduction in large caliber axons in the Dhtkd1 $1^{Y 486^{*} / Y 486^{*}}$ and Dhtkd1 $1^{Y 486^{* /+}}$ mice, with an abundance of smaller caliber axons in comparison to wt mice (Fig. 2g). The number of axons with a large diameter $(>20 \mu \mathrm{m})$ and the thickness of the the myelin sheath $(>2.6 \mu \mathrm{m})$ in the Dhtkd1 $1^{Y 486^{\prime \prime}+}$ and Dhtkd1 $1^{Y 486^{\prime \prime} / Y 486^{\prime \prime}}$ mice were significantly reduced when compare to wt littermates at 8 months of age (Fig. $2 \mathrm{~h}$ and i).

Moreover, behavioral tests demonstrated that Dhtkd1 ${ }^{\mathrm{Y} 486^{*} / Y 486 "}$ mice had a significant longer latency in both the hot plate test (Fig. 2j) and the Von Frey test (Fig. 2k). The increased response latencies indicate a sensory defect, which is in line with the sensory loss in CMT2Q patients [9]. However, no difference between Dhtkd1 $1^{Y 486^{\prime \prime} / Y 486^{\prime \prime}}$ mice and their respective wt littermates was detected in locomotor performance tests, including the treadmill tests (Fig. 2l) and the rotarod test (Fig. 2m). At the histopathological level, no significant difference was observed between mutant and wt mice in the number of the neuromuscular junction as was determined by acetylcholinesterase (AChE) staining (Fig. $2 \mathrm{n}$ and o). When the motor nerve conduction velocity (MNCV) was assessed, no significant difference was detected between the wt and mutant mice (Sup. Figure 3a-b), which would indicate an axonopathy rather than a demyelinating neuropathy. Taken together, the results suggest that alterations in the axons with secondary myelin abnormalities seen in the sciatic nerve were not sufficient to affect either MNCVs or the motor function of the knock-in mice.

\section{Mitochondrial accumulations in gastrocnemius muscle of Dhtkd1 deficient mice}

The biceps brachii muscle biopsy of CMT2Q patients displayed abnormally small and angulated muscle fibers [9]. Based on this observation, we performed histopathology of the gastrocnemius muscle, which is one of the main muscles affected in CMT. Hematoxylin-eosin staining (H\&E) showed a regular size and morphology of the myofibers in both Dhtkd1 ${ }^{Y 486^{\prime \prime} Y 486^{*}}$ and Dhtkd1 $1^{Y 486^{\prime \prime} /+}$ mice at 12 months of age (Fig. 3a). Previously, we reported that silencing of DHTKD1 leads to impaired energy production, evidenced by decreased ATP, total nicotinamide adenine dinucleotide $(\mathrm{NAD}+)$ and $\mathrm{NADH}$, and $\mathrm{NADH}$ levels in vitro [9]. Therefore, we performed a nicotinamide adenine dinucleotide-tetrazolium reductase (NADH-TR), succinate dehydrogenase (SDH) and adenosine triphosphatase
(ATPase) staining in the gastrocnemius muscles (Fig. 3b, c, d). However, we did not observe any reduction of NADHTR and ATPase in Dhtkd1 $1^{Y 486^{\prime \prime} / Y^{486}}$ and Dhtkd1 $1^{Y 486^{\prime \prime}+}$ mice (Fig. 3b, c, d). While the normal morphology and energy status of the gastrocnemius muscles in the Dhtkd1 ${ }^{\text {Y486" }}$ knock-in mouse model does not reproduce the clinical histopathological observations in CMT2Q patients, these results do align with the fact that the mice have no locomotor defects.

Interestingly, when we performed a TEM analysis for the gastrocnemius muscles in the three genotypes of mice at 12 months of age, we observed obvious mitochondrial accumulations in the $\operatorname{Dhtkd1^{Y486^{*}}}$ knock-in mice compared to wt littermates (Fig. 3e). This is supported by the observation that the copy number of mitochondrial DNA in the Dhtkd1 $1^{Y 486^{*} / Y 486^{*}}$ as determined by real-time PCR was significantly increased (Fig. 3f). Morphologically, mitochondria have a larger size in the Dhtkd1 ${ }^{Y 486^{\prime \prime} / Y 486^{\prime \prime}}$ mice compared to the wt mice (Fig. 3e).

\section{Elevated metabolic state may be responsible for the normal locomotor behavior of $\operatorname{Dhtkd} 1^{Y 486^{*} / Y 486^{*}}$ mice}

In order to investigate whether energy metabolism was

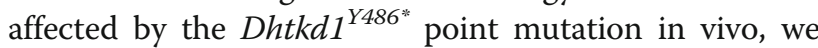

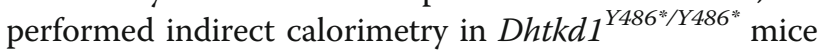
and their wt littermates. Oxygen consumption $\left(\mathrm{VO}_{2}\right)$ and carbon dioxide production $\left(\mathrm{VCO}_{2}\right)$ were measured during $24 \mathrm{~h}$. This revealed that the respiratory exchange ratio (RER, $\mathrm{VCO}_{2} / \mathrm{VO}_{2}$ ) in Dhtkd1 $1^{Y 486^{*} / Y 486^{*}}$ mice (approx. 0.8) was higher than in wt mice (approx. 0.7) continuously along $24 \mathrm{~h}$ (Fig. $4 \mathrm{a}$ and b). This indicates that the Dhtkd1 $1^{Y 486^{*} / Y 486^{*}}$ mice have an elevated metabolic state. As DHTKD1 is presumed to be a nuclearencoded mitochondrial precursor protein, we speculate that this elevated metabolic state in mutant mice could be a consequence of the accumulation of mitochondria [9].

Moreover, using the Affymetrix Mouse Gene 2.0 ST (Affymetrix, Santa Clara, CA) RNA chip, we could detect - at the transcriptional level - an upregulation in glycerolipid metabolic genes in the Dhtkd1 $1^{Y 486^{*} / Y 486^{*}}$ mice in comparison to their wt littermates (Sup. Table 2). The expression of the $D g k g$ gene which encodes diacylglycerol kinase gamma protein was significantly increased in Dhtkd $1^{\text {Y486"Y Y486" }}$ mice (Fig. 4c). Diacylglycerol kinase can phosphorylate diacylglycerol (DAG) to phosphatidic acid (PA) $[15,16]$. The expression of the gene Lipin2, which encodes the lipin2 protein that can turn PA into DAG, was significantly decreased in $D h t k d 1^{Y 486^{*} / Y 486^{*}}$ mice (Fig. 4d). The increase of $D g k g$ and the decrease of Lipin2 resulted mainly in the conversion of DAG. As a consequence, the levels of DAG and triglyceride (TG) are lower and the PA level are higher in blood serum in $D h t k d 1^{Y 486^{*} / Y 486^{*}}$ mice (Fig. 4e). Although the glucose 


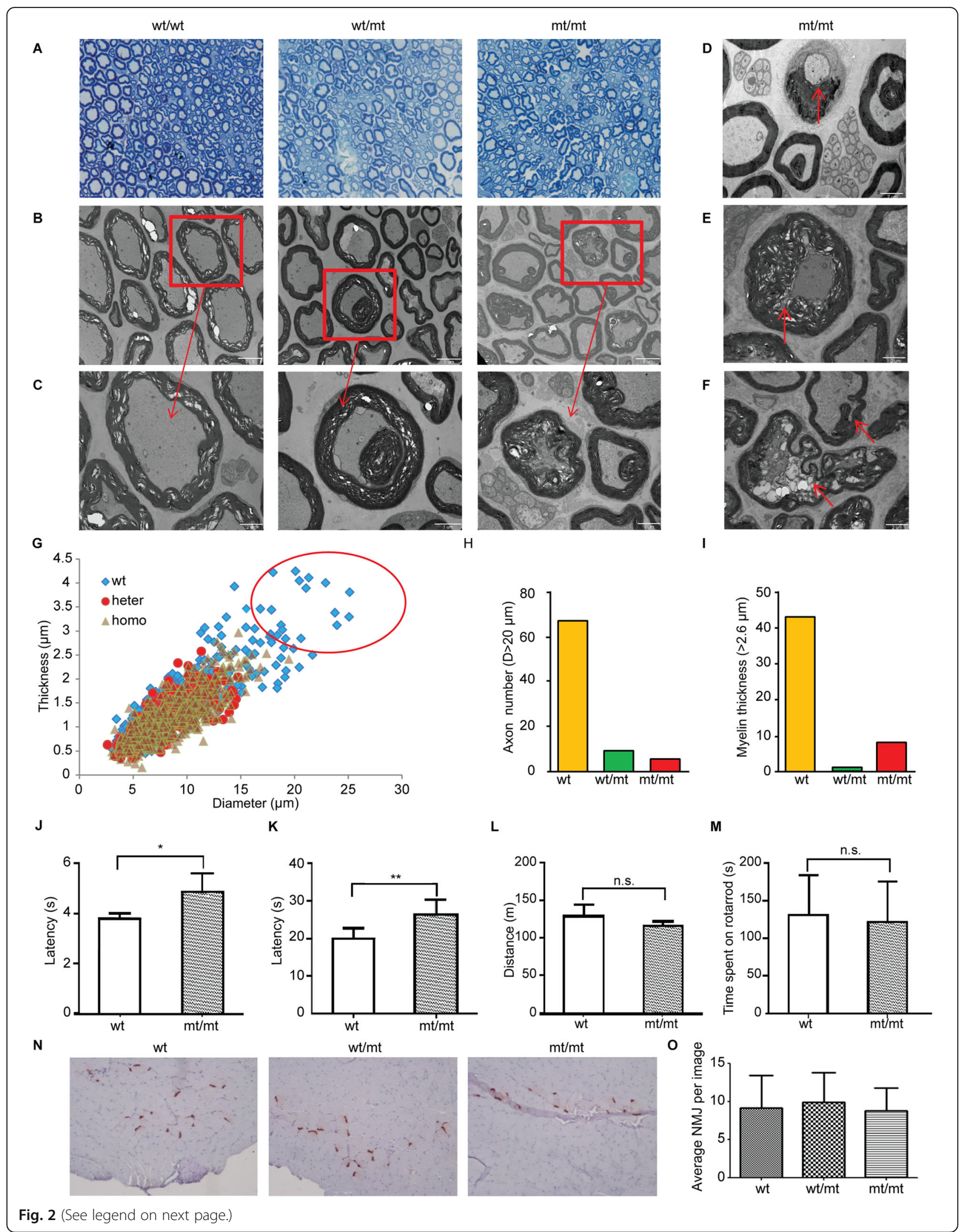


(See figure on previous page.)

Fig. 2 Dhtkd $1^{Y 486^{*}}$ mutant mice exhibited an axonopathy, but no motor defects. a Semi-thin sections of distal sciatic nerve from 8 months old mice were stained with toluidine blue. Images were taken at 1000x magnification. b, c Sections of distal sciatic nerve from 8 month old mice were analyzed using transmission electron microscopy (TEM). Normal and pathological changes of myelin infoldings and axon dissolutions are indicated with arrows in the wt/mt and mt/mt mice. Scale bars: $5 \mu \mathrm{m}$ (b) or $2 \mu \mathrm{m}$ (c) c. d-f Pathological changes of myelin infoldings are indicated with arrows in $\mathrm{mt} / \mathrm{mt}$ mice. Scale bars: $2 \mu \mathrm{m}$. $\mathbf{g}$ The number of large diameter axons $(>20 \mu \mathrm{m})$ and myelin thickness was quantified in the $\mathrm{wt}, \mathrm{wt} / \mathrm{mt}$ and $\mathrm{mt} / \mathrm{mt}$ mice. A significant difference was observed in $\mathrm{wt} / \mathrm{mt}$ and $\mathrm{mt} / \mathrm{mt}$ mice in comparison to the wt group. $\mathbf{h}, \mathbf{i}$ The quantification of axon number (diameter $>20 \mu \mathrm{m}$ ) and number of axons with myelin thickness over $2.6 \mu \mathrm{m}$ in distal sciatic nerve of mice. Ten randomly selected fields (50 100 nerve fibers per field) from 3 to 5 ultrathin cross sections of sciatic nerve per mouse were analyzed, and 3 mice were analyzed per genotype. $\mathbf{j}$ The sensitivity to heat of 8 months old $w t$ and $\mathrm{mt} / \mathrm{mt}$ mice was recorded using the paw pain test $(n=10$; $\left.{ }^{*} p<0.05\right)$. Mt/mt mice displayed a greater latency in retracting their paws in comparison to wt mice. $\mathbf{k}$ The sensitivity threshold to touch of 8 months old wt and $\mathrm{mt} / \mathrm{mt}$ mice was determined using the Von Frey test $\left(n=10\right.$; $\left.{ }^{* *} p<0.01\right)$, which also showed that $\mathrm{mt} / \mathrm{mt}$ mice displayed a greater latency in retracting their paws in comparison to wt mice. I The running distance/min $(\mathrm{m})$ of $8 \mathrm{months}$ old $\mathrm{wt}$ and $\mathrm{mt} / \mathrm{mt} \mathrm{mice}$ was recorded in the treadmill test $(n=10$; n.s. $p>0.05$ ), with no apparent difference between genotypes. $\mathbf{m}$ The time spent on Rotarod by 8 months old wt and $\mathrm{mt} / \mathrm{mt}$ mice was recorded $(n=8 ; p>0.05)$, with no apparent difference between genotypes. $\mathbf{n}$ The morphology of neuromuscular junctions of wt and $\mathrm{mt} / \mathrm{mt}$ mice using AChE staining. Images were taken at 100x magnification. o Quantification of the number of neuromuscular junctions of wt and $\mathrm{mt} / \mathrm{mt}$. AChE staining was performed on frozen sections from the skeletal muscles from 8 months old mice, with no significant difference between genotypes

(GLU) level did not show a difference (Fig. 4f), and the blood lipid level of the high-density lipoproteincholesterol (HDL-C) and the low-density lipoproteincholesterol (LDL-C) showed a decreased trend (Fig. 4g and h). The level of the total cholesterol (TCHO) and triglyceride (TG) were markedly decreased in Dhtkd1 $1^{\text {Y486"/Y486* }}$ mice compared to wt littermates (Fig. $4 j$ and $k$ ).

\section{Discussion}

In this study, we present a new knock-in mouse model for the Dhtkd1 $1^{\text {Y486* }}$ mutation causing CMT2Q. This is the first time that ubiquitous expression of mutant Dhtkd1 is reported at endogenous levels in a mouse model. As the current mouse model harbors the human disease-causing mutation, it was anticipated to be an accurate modelling of the physiological and genetic condition of CMT2Q patients.

Clinically, mutations in CMT-causing genes can result in different phenotypes, ranging from normal to severely disabled, even within the same family. Therefore, it is not surprising that there might be a plethora of genetic and epigenetic factors that modify disease severity in these patients [17]. Moreover, mutations in DHTKD1 are not an exception on this, with patients clinically presenting with a range of neurological conditions to none at all $[18,19]$.

Previously, we reported that the non-sense heterozygous mutation causes a reduction of approximately $50 \%$ of the DHTKD1 expression in the peripheral blood samples of CMT2Q patients [9]. The current mouse model demonstrates also a reduction of Dhtkd1 expression in the Dhtkd1 $1^{Y 486^{\prime \prime}+}$ and $D h t k d 1^{Y 486^{*} / Y 486^{*}}$ mice, which resembles that of the human CMT2Q patients. Furthermore, the $D h t k d 1^{Y 486^{*} / Y 486^{*}}$ mice recapitulate the sensory defects (Fig. 2j, and k), which is a clinical phenotype reported in some CMT2Q patients [9].
The generation of the knock-in mice gives us the opportunity to investigate the pathological changes in peripheral nervous tissue, which clinically has never been done before. An axonopathy in both $\operatorname{Dhtkd1} 1^{Y 486 \%++}$ and Dhtkd1 $1^{Y 486^{*} / Y 486^{*}}$ mice was observed, including a reduction in the number of large caliber axons (Fig. $2 \mathrm{~h}$ and i) and the development of vacuolar inclusions at the axoplasm accompanied with myelin infoldings (Fig. 2f). Additionally, the presence of 'double' myelination of axons by Schwann cells was commonly observed (Fig. $2 b$ and c). Double myelination occurs when outer myelin internodes are maintained despite losing contact with the axon [20]. This typically has been demonstrated during Wallerian degeneration, but it may also be due to retrograde inversion of infolded myelin loops, which has been previously described in a mouse model of CMT4B2 [20,21]. Nerve conduction velocities were normal in $D h t k d 1^{Y 486^{*} / Y 486^{*}}$ mice (Sup. Figure 3), despite a loss of large myelinated axons in the sciatic nerve. However, as the sciatic nerve is mainly composed of myelinated sensory neurons, this may indicate why only a sensory phenotype was detectable in adult mice (Fig. 2j and k) [22]. Therefore, mutations in Dhtkd1 may predominant have pathological effect on the largest metabolically active sensory neurons or Schwann cells or both in our knock-in mouse model (Fig. 2g, h and i).

A possible explanation for the lack of detectable locomotor defects in these knock-in mice may be a metabolicrelated compensatory mechanism that enables maintenance of normal motor functions. The physiological function of DHTKD1 in coding mitochondrial precursor protein led us to check the mitochondrial state in the gastrocnemius muscles. Interestingly, the knock-in mice showed an accumulation of mitochondria in their gastrocnemius muscles (Fig. 3e). It is known that accumulation of damaged mitochondria plays an important landmark of aging and neurodegeneration [23]. However, the accumulated 


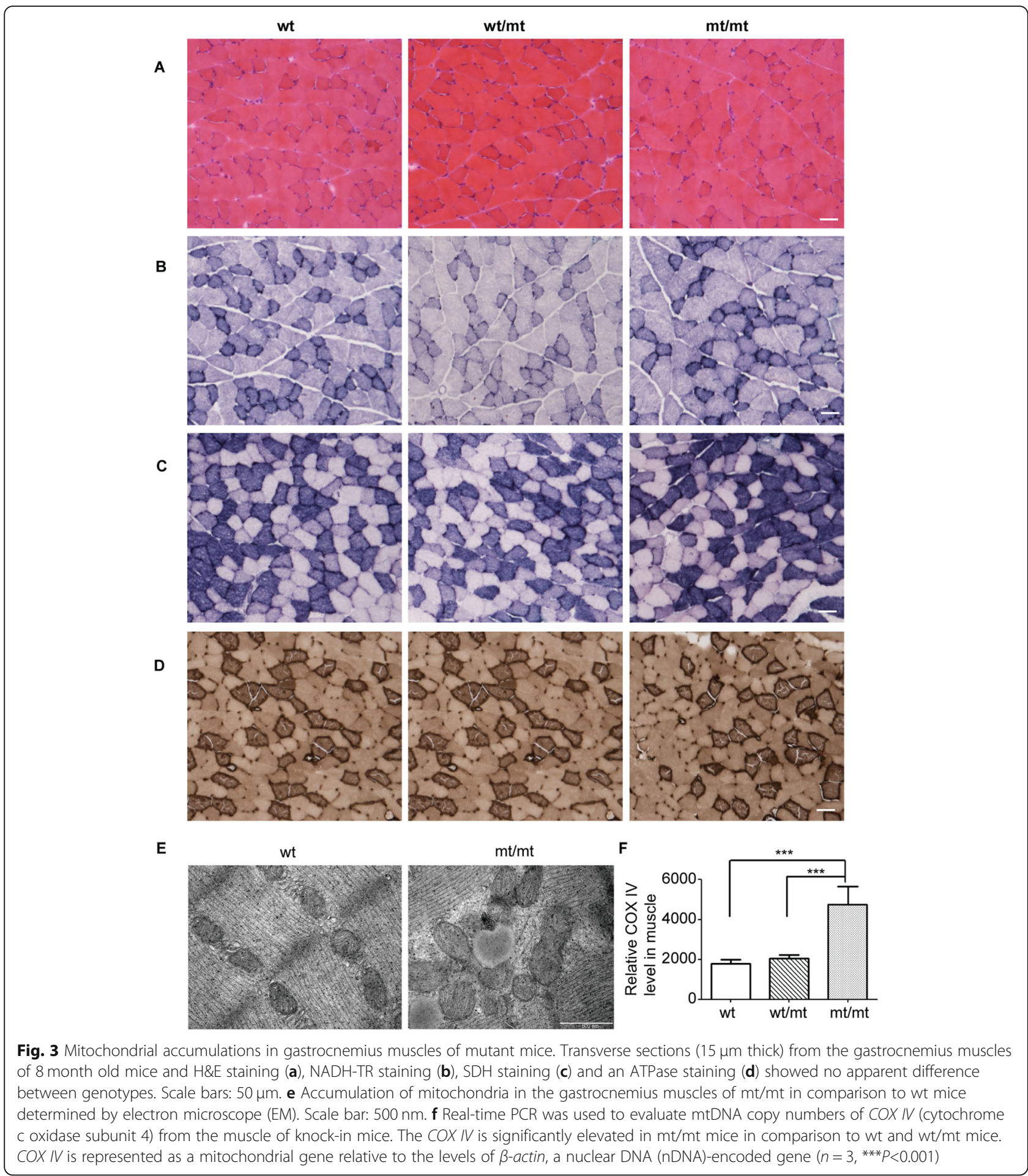

mitochondria in $D h t k d 1^{Y 486^{*} / Y 486^{*}}$ mice did not appear to be damaged. These mitochondria have regular substructures including clear cristae and intramembrane space (Fig. 3e). This indicates that the accumulated mitochondria are still functional. Contradictory to the decreased ATP production caused by knocking down DHTKD1 in vitro, the ATPase staining in gastrocnemius muscles of
$D h t k d 1^{Y 486^{*} / Y 486^{*}}$ mice did not show any differences between wt and knock-in mice, which implies that the mitochondria were functional (Fig. 3d) [9]. Combined with the fact that the expression level of Dhtkd1 was remarkably decreased in knock-in mice, we assume that increased mitochondrial biogenesis compensates for the insufficient nuclear coded mitochondrial precursors caused 


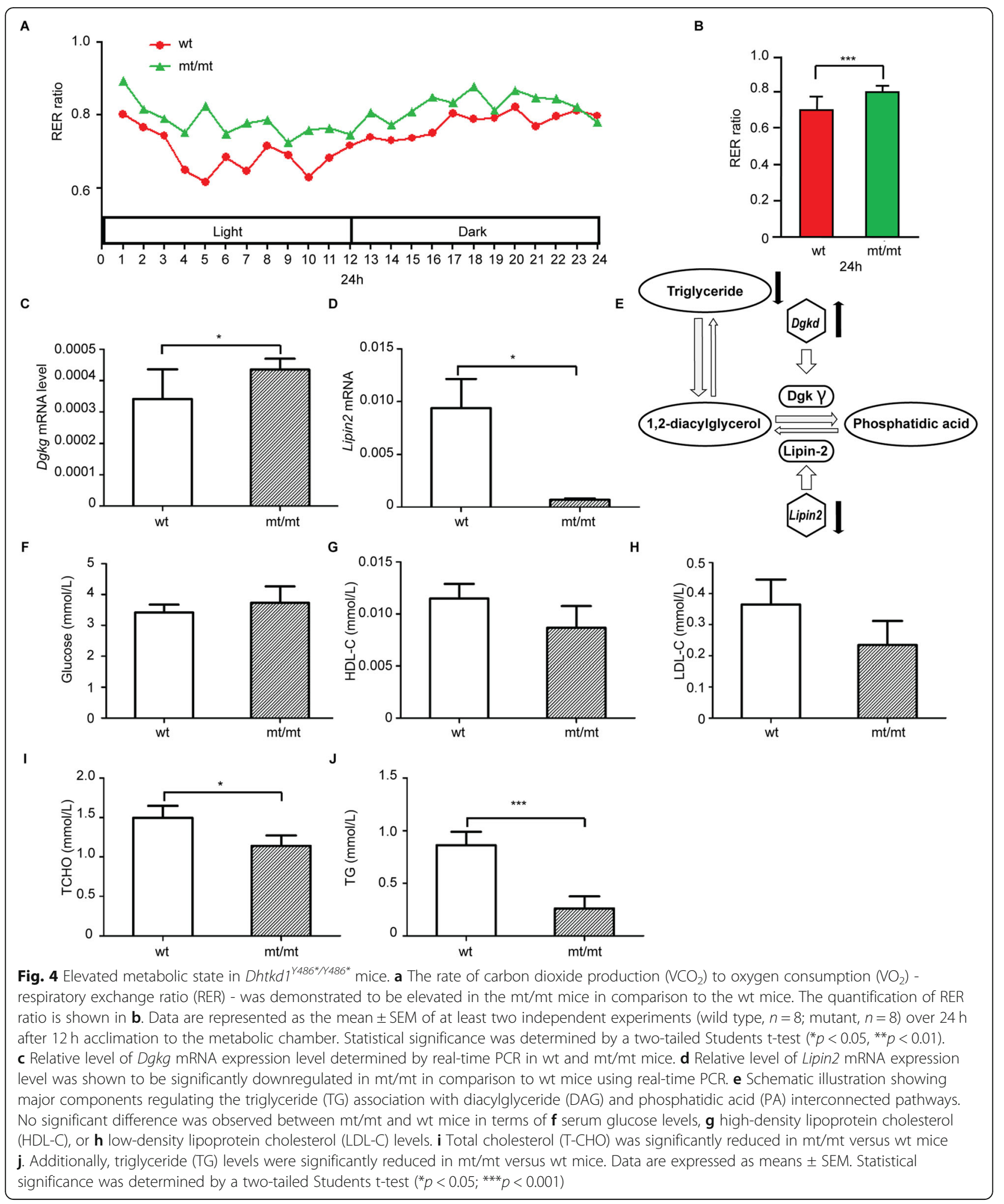

by the non-sense mutation. Thereby, the mitochondrial metabolic capacity remains normal and eventually supports the motor functions in our knock-in mice. This is also in line with the fact that patients with 2-aminoadipic and 2-oxoadipic aciduria related biallelic mutations in DHTKD1 are only mildly symptomatic or are asymptomatic. As a consequence, compensatory mechanisms might also apply to human [18]. 
Moreover, the overall energy metabolism of $D h t k d 1^{Y 486^{*} / Y 486^{*}}$ mice appears elevated in comparison to wt mice based on the indirect calorimetry assay (Fig. 4a and b). The apparent increase in metabolism may compensate for the locomotor defects in adult knock-in mice. Recently, altering the metabolic state in CMT rodent models has been targeted as a therapeutic intervention. Lipid metabolism in peripheral nerves was demonstrated to be downregulated in a rat model of CMT1A - the most common form of CMT - and using a high lipid diet, it was shown to mitigate disease pathogenesis [24]. In addition, the ketogenic diet - a high fat and low carbohydrate diet - has been shown to be beneficial in other myelinopathies, such as multiple sclerosis and Pelizaeus-Merzbacher disease $[25,26]$. As a consequence, the metabolic state of peripheral tissue can strongly influence the phenotype in rodent models, as well as in patients.

In conclusion, we show that the Dhtkd1 ${ }^{Y 486^{*}}$ knock-in mouse model partially recapitulates clinical phenotypes of CMT2Q patients, especially in relation to the sensory deficit and axonopathy. We hypothesize that the lack of locomotor defects might be due to a mechanism that compensates for the defects in energy metabolism. Lastly, as altered metabolic states in peripheral neuropathies are becoming more apparent as a cause or consequence of the genetic mutation, targeting metabolism through dietary changes may be beneficial for the current mouse model and possibly also for patients.

\section{Materials and methods}

\section{Construction of Dhtkd $1^{\text {Y486* }}$ knock-in targeting vector}

The Dhtkd1 targeting vector was obtained by the Red/ ET cloning method [16]. Briefly, homology arms (A, B, C, D) were amplified from a BAC (Bacterial Artificial Chromosome) clone containing the Dhtkd1 genomic sequence. Primers were as follows:

A1: TTTAAGCTTGGCTGTGTTCACTCATCACATT, and

A2: ATGCTCGAGTGCTACTGTTTCGTGGTACCTT,

B1: ATGCTCGAGGTGCAGAGGAAGCCAACACT, and

B2: ACAAGGATCCTCTTTACCTCCCTGGTGCTG,

C1: TTTCCGCGGAAAGCAGTTGGGTGCTCTTACC, and

C2: ACAAGGATCCTAGGTGACCCCTGTGAAAAG GT,

D1: TTTAAGCTTACAAACATGAAGCAGCAACAGA, and

D2: TACGGGTACCCTGGCATCCTCACAGACATA CA.

PCR products A and B were digested by HindIII / XhoI and XhoI/BamHI, respectively, and inserted into HindIII BamHI site of pBR322-MK-MCS to produce the retrieving vector pBR322-MK-MCS-AB. After linearization by XhoI, genomic DNA containing Dhtkd1 was retrieved from the BAC clone and inserted into the vector. A site-directed mutagenesis procedure was used to introduce the $D h t k d 1^{Y 486^{*}}$ point mutation into the vector by using two partly complimentary oligonucleotides with the following sequence:

Dhtkd1 mut-1: CATTTAACTTGGTCTAGTAGGA GGTTTTTATGTCGG;

Dhtkd1 mut-2: AACCTCCTACTAGACCAAGTTA AATGACCACTTGG.

The resulting mutated DNA fragment was verified by Sanger sequencing. Similarly, PCR products C and D were digested by SacII/BamHI and HindIII/Kpn I, respectively, and ligated to Sac II/BamH I and Hind III/ Kpn I sites of the PL451 vector containing the PGK-neo cassette. C-neo-D fragment was amplified from the vector using primers $\mathrm{C} 1$ and $\mathrm{D} 2$, and cloned into pBR322MK-MCS-AB to obtain the targeting vector.

\section{Generation of the Dhtkd ${ }^{\text {Y486* }}$ knock-in mice}

The targeting construct was linearized with Not I and electroporated into SCR012 embryonic stem (ES) cells. Resistant cells were selected in the presence of G418 (G418 sulfate; Geneticin; Gibco) and Ganciclovir. DNA was isolated from a total of 96 clones and analyzed by PCR to identify positive clones that had undergone homologous recombination with the targeting vector. Primers for evaluation of the $5^{\prime}$ arm and the $3^{\prime}$ arm were P1 (5' ${ }^{\prime}$ TCATGC AGTTGGTGCGATA-3') and P2 (5'- AGACAATCGG CTGCTCTGAT-3'); P3 (5'-TACCCGGTAGAATTTCGA CGA-3') and P4 (5'-TGGAGATGGGAGGACATACA CT-3'), giving products with a length of $5040 \mathrm{bp}$ and 4414 bp, respectively.

One ES cell line was injected into C57BL/6 J blastocysts, which were subsequently transferred into pseudopregnant females to generate chimeric offspring. Chimeras were bred with $\mathrm{C} 57 \mathrm{BL} / 6 \mathrm{~J}$ female mice to produce heterozygous mice. The homozygous mice with mutant gene were obtained by crossing between heterozygous mice. All mice were housed in a temperaturecontrolled facility in rooms maintained on a $12 \mathrm{~h}$ light/12 h dark cycle, with free access to regular chow and water.

\section{Genotyping of mutant mice}

The genotype of the mutant mice was determined by PCR of genomic DNA extracted from mouse tail with specific primers for the WT allele (P7: $5^{\prime}$ - CCCGTC TCTATTTTCCACCA - 3' and P8: 5' - ATCTCTGTGG CTTTGGTGGTC $-3^{\prime}$ ) and for the targeted allele (P7 and P2). The three primers were used in a multiplex PCR with La Taq (Takara). The amplification conditions were as follows: $95^{\circ} \mathrm{C}$ for $5 \mathrm{~min}$ and 35 cycles of $95^{\circ} \mathrm{C}$ for $30 \mathrm{~s}, 58^{\circ} \mathrm{C}$ for $30 \mathrm{~s}, 72{ }^{\circ} \mathrm{C}$ for $2.5 \mathrm{~min}$, and a $10 \mathrm{~min}$ 
incubation at $72{ }^{\circ} \mathrm{C}$ at the end of the run. PCR products were seperated on a $1.0 \%$ agarose gel. Product length of WT and targeted allele were $1355 \mathrm{bp}$ and $2462 \mathrm{bp}$, respectively.

\section{RT-PCR and real-time PCR}

Total RNA was extracted using TRIzol Reagent (Life Technologies Inc., Gaithersburg, MD, USA) according to the manufacturer's instructions. The first-strand cDNAs were synthesized from total RNA with oligo (dT) primers and random 9-mer primers using PrimerScript TM RTase (Takara, Dalian, China) at $37^{\circ} \mathrm{C}$ for $15 \mathrm{~min}$. One microliter of the reverse transcription reaction products was used as PCR template. Dhtkd1 expression was detected by semi-quantitative RT-PCR using as primers: 5'-ATATCAGCGCCAGTTCCG-3' and 5'TGAGGTGCTCTGCGTAGGT-3'. Real-time PCR was carried out using SYBR ${ }^{\circ}$ Premix Ex Taq ${ }^{\text {tix }}$ (Takara) in 96well optical reaction plates on an ABI PRISM 7900 HT Real-time PCR system according to the manufacturer's protocol. The cycle threshold (CT) values of Dhtkd1 primers were compared with those of Gapdh-specific primers using the comparative CT method. The primers used in real-time PCR were the following:

Dhtkd1 sense, 5'-GGTGCAGCCAGAAGCATG-3', Dhtkd1 anti-sense, 5' -GGAGCCCAAGGCAAGTGT-3', Gapdh sense, 5'-CCTCGTCCCGTAGACAAAATGGT-3', Gapdh anti-sense, 5'-TTGAGGTCAATGAAGGGG TCGT-3'.

\section{Western blot analysis}

Proteins were extracted from mouse kidney and the concentration was determined using the BCA Protein Assay Kit (Beyotime, China). Equal amounts of proteins were separated by $8 \%$ SDS-PAGE and transferred to nitrocellulose membranes (catalog no. 162-0112; Whatman, UK). Membranes were blocked with 5\% non-fat milk for $1 \mathrm{~h}$, followed by incubation with the primary antibodies as indicated overnight at $4{ }^{\circ} \mathrm{C}$. Antibodies used were a goat polyclonal antibody raised against Dhtkd1 (1:200 dilution; code sc-242576; Santa Cruz Biotechnology USA), a mouse monoclonal antibody against $\alpha$-tubulin (1:1000 dilution; code sAT819; Beyotime, China). Infrared fluorescence on membranes was detected by using the Odyssey infrared imaging system (LI-COR Biotechnology, Nebraska, USA).

\section{Differentially expressed genes analysis}

RNA chip from the Affymetrix Mouse Gene 2.0 ST (Affymetrix, Santa Clara, CA) was performed by GeneTech (Shanghai, China) to detect differentially expressed genes between wild-type mice and $\mathrm{mt} / \mathrm{mt}$ mice. The differentially expressed genes were mapped to KEGG (http://www.genome.jp/kegg/) pathways to analyse the function of these genes.

\section{ATP assay}

The levels of ATP were measured using an ATP assay kit (Abnova, Taiwan) according to the manufacturer's instructions. Briefly, mouse liver and ES cells were lysed in $100 \mu \mathrm{l}$ of ATP buffer, and then centrifuged in ice-cold buffer at $15,000 \mathrm{~g}$ for $2 \mathrm{~min}$. Supernatant was collected and $50 \mu \mathrm{l}$ was added to a 96-well plate. Subsequently, reaction mix $(50 \mu \mathrm{l})$ was added to each well before incubating at room temperature for $30 \mathrm{~min}$ in the dark. A standard curve was generated using different ATP concentrations $(0,2,4,6,8,10 \mathrm{nmol} /$ well $)$. In the ATP colorimetric assay, O.D. was measured at $570 \mathrm{~nm}$. Total ATP levels were expressed as $\mathrm{nmol} / \mu \mathrm{l}$.

\section{$\mathrm{NADP}^{+} / \mathrm{NADPH}$ assay}

Quantification of $\mathrm{NADP}^{+} / \mathrm{NADPH}$ was performed using a NADP ${ }^{+} / \mathrm{NADPH}$ Quantification kit (BioVision, Zurich, Switzerland) according to the manufacturer's instructions. Briefly, mice liver and ES cells were extracted with 200-400 $\mu \mathrm{l} \mathrm{NADP} / \mathrm{NADPH}$ extraction buffer. Subsequently, the sample was centrifuged at $14,000 \mathrm{~g}$ for 5 min. To measure total $\mathrm{NADP}^{+} / \mathrm{NADPH}$ (NADPt), $50 \mu \mathrm{l}$ of extracted samples was transferred in duplicate into a 96-well plate. Samples were heated to $60^{\circ} \mathrm{C}$ for $30 \mathrm{~min}$ in a water bath. Subsequently, reaction mix $(100 \mu \mathrm{l})$ was added to each well. The plates were incubated at room temperature for $5 \mathrm{~min}$. Subsequently, NADPH developer $(10 \mu \mathrm{l})$ was added to each well and the reaction was developed for $4 \mathrm{~h}$. In the $\mathrm{NADP}^{+} / \mathrm{NADPH}$ colorimetric assay, O.D. was measured at $450 \mathrm{~nm}$.

\section{Measurement of mtDNA copy numbers}

The mtDNA copy number was detected by real-time PCR which was performed with a Mastercycler ep realplex (Eppendorf) using the SYBR Premix Ex Taq ${ }^{\mathrm{Tm}}$ (Takara). The special primers were GAPDH forward and GAPDH reverse for GAPDH; and cytochrome c I up and cytochrome c I lower for cytochrome oxidase c subunit I (COI) in real-time PCR assay. A total of $25 \mathrm{ng}$ of DNA was used and the number of PCR cycles to reach this amount of DNA was defined as the threshold cycle value $(\mathrm{Ct})$. The copy number of mtDNA was normalized to the GAPDH content.

\section{Blood lipid assay}

Wt mice and $\mathrm{mt} / \mathrm{mt}$ mice ( $n=12$ for each) were deprived of food and water $8 \mathrm{~h}$ before the assay was performed. Blood sample were drawn from retrobulbar blood vessels, centrifuged at $3000 \mathrm{~g}$ for $10 \mathrm{~min}$ and the upper liquid was transferred to a new centrifuge tube and was considered as serum. Blood lipid assays were 
performed by Shanghai Research Centre for Model Organisms, Shanghai, China.

\section{Histological analysis}

Sciatic nerves were fixed by transcardial perfusion with $4 \%$ paraformaldehyde, dissected and post-fixed overnight in the same fixative. Tissue was then processed for plastic embedding and transmission electron microscopy (TEM) by standard procedures. For nerve histology, $0.5 \mu \mathrm{m}$ sections were stained with Toluidine blue and examined by light microscopy. TEM images were collected on a Jeol 1230 transmission electron microscope. Axon diameter was determined from 5 non-overlapping fields from each of three mutant and three littermate control samples. Distances were determined using the associated software. For counts of axons, left and right nerves were taken whenever possible, and counts were averaged so that each nerve represents one mouse, which is the average count of the left and right nerve. The total number of myelinated axons in each nerve was counted using light microscopy on Toluidine blue stained sections.

For muscle analysis, dissected gastrocnemius muscle were washed briefly in PBS to remove as much blood as possible, then were fixed in $10 \%$ neutral buffered formalin, embedded in paraffin blocks, sectioned (5um) transversely and stained with haematoxylin and eosin (H\&E). H\&E staining was performed by incubating sections in $1 \%$ formol-calcium (10 min), in Harris' haemtoxylin (3 min; Sigma Aldrich), in water (3 min) and in eosin (3 min). For enzyme staining, the gastrocnemius muscle was dissected and frozen fresh in isopentane supercooled by liquid nitrogen. Ten-micrometer frozen transverse sections were cut with a cryostat (Leica CM 1850) AChE staining was performed by using a staining kit (DE0056) from Leagene Biotechnology (Beijing, China). The AChE incubating solution is prepared based on the protocol of the kit and the incubation time is less than $6 \mathrm{~h}$ in dark till grey color appear. The AChE activities are recognized based on the red to grey staining spots. Staining for Nicotinamide adenine dinucleotide (NADH), succinate dehydrogenase (SDH) and myosin ATPase (PH 4.3) using standard methods. For NADH staining, the procedures are: 1 . Muscle tissue placed in a staining tray in a damp atmosphere; 2. Drop a few incubating solution (contain $30 \mathrm{ml} 0.05 \mathrm{M}$ Tris buffer, $30 \mathrm{mg}$ nitro blue tetrazolium and $24 \mathrm{mg} \beta-\mathrm{NADH}, \mathrm{PH7}$.4) to fully cover the section; 3. Incubate for $30 \mathrm{~min}$ at $37^{\circ} \mathrm{C}$; 4 . Rinse in distilled water and mount in aqueous mountant. For $\mathrm{SDH}$ staining, the procedures are similar as NADH, while the incubating solution contains $15 \mathrm{ml} 0.2 \mathrm{M}$ Sodium succinate, $15 \mathrm{ml} 0.2 \mathrm{M}$ phosphate buffer ( $\mathrm{pH}$ 7.4) and $30 \mathrm{mg}$ Nitro blue tetrazolium. For both NADH and SDH staining, the incubating solution contains nitro blue tetrazolium, which gives a blue product with
$\mathrm{NADH}$ or SDH activity. For ATPase staining, the procedures are: 1 . Place one coverslip for each biopsy in a separate, labeled staining jar for incubating with 4.3 ATP solution ( $5 \mathrm{ml}$ Barbital Acetate Solution, $10 \mathrm{ml} 0.1 \mathrm{~N}$ $\mathrm{HCl}$ and $8 \mathrm{ml}$ deionized water, $\mathrm{PH} \mathrm{4.3)} \mathrm{five} \mathrm{minutes} \mathrm{at}$ room temperature and pour out the solution; 2. Pour the washing solution (contain $6 \mathrm{ml} \mathrm{0.1} \mathrm{M} \mathrm{Sodium} \mathrm{Barbi-}$ tal, $3 \mathrm{ml} 0.18 \mathrm{M}$ Calcium Chloride and $21 \mathrm{ml}$ deionized water, $\mathrm{PH}$ 9.4-9.7) into the staining jar for $30 \mathrm{~s}-1 \mathrm{~min}$ and pour out the solution; 3. Pour the ATP solution (contain $75 \mathrm{mg}$ ATP powder, $6 \mathrm{ml} 0.1 \mathrm{M}$ Sodium Barbital, $21 \mathrm{ml}$ deionized water and $3 \mathrm{ml} 0.18 \mathrm{M} \mathrm{Cal-}$ cium Chloride, $\mathrm{PH}$ 9.4-9.7) into the staining jar for 45 min at $37^{\circ} \mathrm{C} ; 4$. Wash the staining jar with three changes of $1 \%$ Calcium Chloride for three rinses, $3 \mathrm{~min}$ each; 5. Wash the staining jar with three changes of $2 \%$ Cobalt Chloride for three rinses, 3 min each. 6. Add 1\% ammonium sulfide to the staining jar for at least 1-3 min; 7. Rinse in the fume hood with approximately 3-5 changes of tap water; 8 . Dehydrate in ascending alcohols and clear with at least two changes of xylene; 9. Mount coverslips onto labeled glass slides with Canda Balsam. All photomicrographs were taken on a Nikon N80i microscope. Images were taken under 200x magnification.

For electron microscopy, the gastrocnemius muscles were dissected, cut into $1 \mathrm{~mm}$ cubes, fixed in $1 \%$ glutaraldehyde in PBS at $4{ }^{\circ} \mathrm{C}$ overnight, and further processed for staining, embedding, sectioning and post-staining. Specimens were then examined and photographed with an electron microscope (Philips CM10).

\section{Behavioral tests}

The paw pain test and the Von Frey test were both used to measure sensory abnormalities. For paw pain test, wt and $\mathrm{mt} / \mathrm{mt}$ mice were fixed in the fixator and only mice's hind paws were exposed to the infrared heat source $(30 \mathrm{U})$. The latency until the animal moved its hind paws away from the heat source was recorded. For Von Frey test, the wt and $\mathrm{mt} / \mathrm{mt}$ mice stood on an elevated platform in which the surface was a wide gauge wire mesh. Von-Frey hair was inserted from below, up through the holes in the mesh, to poke the undersurface of a hind paw. At threshold, the mouse's responds by quickly flicking its hind paw away from the hair. Mechanical withdrawal threshold was defined as the minimum gauge wire stimulus that elicited withdrawal reaction.

The treadmill test and the rotarod tests were used to measure motor performance. For the rotarod test, wt and $\mathrm{mt} / \mathrm{mt}$ mice were placed on a standard rotarod apparatus. The latency for a mouse to fall off the accelerating rotarod $(20 \mathrm{rpm})$ was recorded. For treadmill test, made of a continuous belt ( $40 \mathrm{~cm}$ long and $14 \mathrm{~cm}$ wide), operating at an adjustable speed $(\mathrm{v}=10 \mathrm{~m} / \mathrm{min})$. A grid 
at the end of the belt administered an electric shock to force the mouse to run. The time spent on the grid was recorded by a counter.

\section{Indirect calorimetry}

Wt or $D h t k d 1^{-/-}$male mice (5 6 mouth old) were housed individually in metabolic cages (TES LabMaster system) for $72 \mathrm{~h}$ according to the instructions of the manufacturer. After the mice adapted to the metabolic cages for $12 \mathrm{~h}$, volume of food consumed, total movement distance, $\mathrm{O}_{2}$ consumption and $\mathrm{CO}_{2}$ production were continuously recorded over $24 \mathrm{~h}$ periods.

\section{Statistical analysis}

Data were represented as mean \pm standard deviation (SD) as indicated. Statistical significance between any 2 groups was determined by a 2-tailed Student $t$ test. Multiple group analyses were performed by ANOVA. $p$ values less than 0.05 were considered as significant.

\section{Supplementary information}

Supplementary information accompanies this paper at https://doi.org/10. 1186/s40478-020-00901-0.

Additional file 1: Figure S1. Weight curves. Weight of mice belonging to the three genotypes is shown for the male (A) and female (B) mice as a function of the age of the mice. $X$-axis represents month. The number of mice is 10 in three genotypes. Figure S2. Expression profile of Dhtkd1 gene in adult mice. Tissue-specific expression levels of Dhtkd1 mRNA were examined in major tissues of normal adult mice using real-time quantitative PCR. The results are from two independent experiments and each sample was analyzed in triplicate. Figure S3. Motor nerve conduction velocity (MNCV) and sensory nerve conduction velocity (SNCV) in wild type and gene-modified mice. (A) MNCV between WT and HOMO. (B) SNCV between WT and HOMO. Table S1. Dhtkd1 ${ }^{\text {Y }^{4} 6^{*}}$ mutation does not change Mendelian segregation ratio. Dhtkd1 mutant homozygous mice were obtained by crossbreeding heterozygous mice. Statistical analysis included the number of $w \mathrm{t}, \mathrm{wt} / \mathrm{mt}$ and $\mathrm{mt} / \mathrm{mt}$ mice. Table $\mathbf{S 2}$. Pathway analysis of differentially expressed gene.

\section{Acknowledgments}

We thank Shun-yuan Lu, Hong-xin Zhang, Ling-yun Tang, Wang-yang Xu and Jian Fei for technical assistance.

\section{Authors' contributions}

CJ. L and W. G planned and performed most of the experiments. CJ. L coordinate the consistency of the experiments. L. C, XW. W and YM. H helped with most of the experiments. Y. C and SY. D helped with construction of this mouse model and cell culture. Y. K helped with construction of mouse model. X. L monitored the histological experiment and helped with improving the manuscript. ZG. W, L.V.D.B and R. P. helped with improving manuscript. ZG. W and MM. G planned and supervised the project. R.P., W. G and MM. G wrote the manuscript. All authors read and approved the final manuscript.

\section{Funding}

This work was partially supported by grants from National Natural Science Foundation of China (31571295 to M.M.G.), the Ministry of Science and Technology of China (2011BAI15B02 to Z.G.W.) and the E-Institutes of Shanghai Municipal Education Commission (E03003 to Z.G.W. and M.M.G.).

\section{Availability of data and materials}

The datasets during and/or analysed during the current study available from the corresponding author on reasonable request.

Ethics approval and consent to participate

All research protocols involving animal experiments were approved by the Institutional Animal Care and Use Committee of the Shanghai Research Center for Model Organisms or by the Animal Use and Care Committee of the Shanghai Jiao Tong University School of Medicine.

\section{Competing interests}

The authors declare that they have no competing interests.

\section{Author details}

${ }^{1}$ Department of Medical Genetics, E-Institutes of Shanghai Universities, Shanghai Jiao Tong University School of Medicine (SJTUSM), 280 South Chongqing Road, Shanghai 200025, People's Republic of China. ${ }^{2}$ Department of Neurosciences, Experimental Neurology and Leuven Brain Institute (LBI), KU Leuven, University of Leuven, Leuven, Belgium. ${ }^{3} \mathrm{VIB}$, Center for Brain \& Disease Research, Laboratory of Neurobiology, Leuven, Belgium.

${ }^{4}$ Department of Neurology, Children's Hospital of Fudan University, Shanghai, China. ${ }^{5}$ Shanghai Research Centre for Model Organisms, Shanghai, China.

${ }^{6}$ Research Center for Experimental Medicine, Rui-Jin Hospital at SJTUSM, Shanghai, China

Received: 17 January 2020 Accepted: 21 February 2020

Published online: 13 March 2020

\section{References}

1. Tooth HH (1888) The Peroneal Type of Progressive Muscular Atrophy. Ann Surg 7:293 Available from: https://insights.ovid.com/crossref?an=00000658-1 88801000-00057

2. Lupski JR, Reid JG, Gonzaga-Jauregui C, Rio Deiros D, Chen DCY, Nazareth L et al (2010) Whole-genome sequencing in a patient with Charcot-MarieTooth neuropathy. N Engl J Med 362:1181-1191 Available from: http:// www.nejm.org/doi/abs/10.1056/NEJMoa0908094

3. Dyck PJ (1968) Lower Motor and Primary Sensory Neuron Diseases With Peroneal Muscular Atrophy. Arch Neurol 18:603 Available from: http:// archneur.jamanetwork.com/article.aspx?doi=10.1001/archneur.1968.0047036 0025002

4. Timmerman V, Strickland A, Züchner S (2014) Genetics of Charcot-MarieTooth (CMT) Disease within the Frame of the Human Genome Project Success. Genes (Basel) 5:13-32 Available from: http://www.mdpi.com/2 073-4425/5/1/13

5. d'Ydewalle C, Krishnan J, Chiheb DM, Van Damme P, Irobi J, Kozikowski AP et al (2011) HDAC6 inhibitors reverse axonal loss in a mouse model of mutant HSPB1-induced Charcot-Marie-Tooth disease. Nat Med 17:968-974. https://doi.org/10.1038/nm.2396

6. Prior R, Van Helleputte L, Benoy V, Van Den Bosch L (2017) Defective axonal transport: A common pathological mechanism in inherited and acquired peripheral neuropathies. Neurobiol Dis 105:300-320 Available from: https:// linkinghub.elsevier.com/retrieve/pii/S0969996117300323

7. Jerath NU, Shy ME (2015) Hereditary motor and sensory neuropathies: Understanding molecular pathogenesis could lead to future treatment strategies. Biochim Biophys Acta - Mol Basis Dis 1852:667-678 Available from: https://linkinghub.elsevier.com/retrieve/pii/S092544391400252X

8. Rossor AM, Polke JM, Houlden H, Reilly MM (2013) Clinical implications of genetic advances in Charcot-Marie-Tooth disease. Nat Rev Neurol 9:562571 Available from: http://www.nature.com/articles/nrneurol.2013.179

9. Xu W, Gu M, Sun L, Guo W, Zhu H, Ma J et al (2012) A nonsense mutation in DHTKD1 causes Charcot-Marie-Tooth disease type 2 in a large Chinese pedigree. Am J Hum Genet 91:1088-1094 Available from: https://linkinghub. elsevier.com/retrieve/pii/S0002929712005277

10. Bunik VI, Degtyarev D (2008) Structure-function relationships in the 2-oxo acid dehydrogenase family: substrate-specific signatures and functional predictions for the 2-oxoglutarate dehydrogenase-like proteins. Proteins Struct Funct Bioinforma 71:874-890. https://doi.org/10.1002/prot.21766

11. Bunik VI, Fernie AR (2009) Metabolic control exerted by the 2-oxoglutarate dehydrogenase reaction: a cross-kingdom comparison of the crossroad between energy production and nitrogen assimilation. Biochem J 422 
:405-21. Available from: https:/portlandpress.com/biochemj/article/422/3/4 05/44984/Metabolic-control-exerted-by-the-2oxoglutarate

12. Xu W, Zhu H, Gu M, Luo Q, Ding J, Yao Y et al (2013) DHTKD1 is essential for mitochondrial biogenesis and function maintenance. FEBS Lett 587: 3587-3592. https://doi.org/10.1016/j.febslet.2013.08.047

13. Liu P (2003) A Highly Efficient Recombineering-Based Method for Generating Conditional Knockout Mutations. Genome Res 13:476-484 Available from: http://www.genome.org/cgi/doi/10.1101/gr.749203

14. Savastano LE, Laurito SR, Fitt MR, Rasmussen JA, Gonzalez Polo V, Patterson SI (2014) Sciatic nerve injury: a simple and subtle model for investigating many aspects of nervous system damage and recovery. J Neurosci Methods 227:166-180 Available from: https://inkinghub.elsevier.com/retrieve/pii/ S0165027014000314

15. Topham MK, Prescott SM (1999) Mammalian Diacylglycerol kinases, a family of lipid kinases with signaling functions. J Biol Chem 274:11447-11450 https://doi.org/10.1074/jbc.274.17.11447

16. Ferguson PJ (2005) Homozygous mutations in LPIN2 are responsible for the syndrome of chronic recurrent multifocal osteomyelitis and congenital dyserythropoietic anaemia (Majeed syndrome). J Med Genet 42:551-557 Available from: http://jmg.bmj.com/cgi/doi/10.1136/jmg.2005.030759

17. Garcia CA, Malamut RE, England JD, Parry GS, Liu P, Lupski JR (1995) Clinical variability in two pairs of identical twins with the Charcot-Marie-Tooth disease type 1A duplication. Neurol Int 45:2090-2093 Available from: http:// www.neurology.org/cgi/doi/10.1212/WNL.45.11.2090

18. Danhauser K, Sauer SW, Haack TB, Wieland T, Staufner C, Graf E et al (2012) DHTKD1 mutations cause 2-Aminoadipic and 2-Oxoadipic Aciduria. Am J Hum Genet 91:1082-1087 Available from: https://linkinghub.elsevier.com/ retrieve/pii/S0002929712005289

19. Stiles AR, Venturoni L, Mucci G, Elbalalesy N, Woontner M, Goodman S et al (2015) New Cases of DHTKD1 Mutations in Patients with 2-Ketoadipic Aciduria. JIMD Rep 25:15-19 Available from: http://link.springer.com/10.1 007/8904_2015_462 https://doi.org/10.1007/8904_2015_462.

20. Heath JW, Kidd GJ, Trapp BD, Dunkley PR (1991) Myelin maintenance by Schwann cells in the absence of axons. Neurosci Lett 128:277-280 Available from: https://linkinghub.elsevier.com/retrieve/pii/0304394091902793

21. Tersar K, Boentert M, Berger P, Bonneick S, Wessig C, Toyka KV et al (2007) Mtmr13/Sbf2-deficient mice: an animal model for CMT4B2. Hum Mol Genet 16:2991-3001 Available from: https://academic.oup.com/hmg/articlelookup/doi/10.1093/hmg/ddm257

22. Schmalbruch $\mathrm{H}$ (1986) Fiber composition of the rat sciatic nerve. Anat rec 215:71-81 Available from: https://www.ncbinlm.nih.gov/pubmed/18086465 https://doi.org/10.1002/ar.1092150111

23. Tatsuta T, Langer $T$ (2008) Quality control of mitochondria: protection against neurodegeneration and ageing. EMBO J 27:306-314 Available from: http://emboj.embopress.org/cgi/doi/10.1038/sj.emboj.7601972

24. Fledrich R, Abdelaal T, Rasch L, Bansal V, Schütza V, Brügger B et al (2018) Targeting myelin lipid metabolism as a potential therapeutic strategy in a model of CMT1A neuropathy. Nat Commun 9:3025 Available from: http://www.nature.com/articles/s41467-018-05420-0

25. Brenton JN, Banwell B, Bergqvist AGC, Lehner-Gulotta D, Gampper L, Leytham E et al (2019) Pilot study of a ketogenic diet in relapsing-remitting MS. Neurol - Neuroimmunol Neuroinflammation 6:e565 Available from: http://nn.neurology.org/lookup/doi/10.1212/NXI.0000000000000565

26. Stumpf SK, Berghoff SA, Trevisiol A, Spieth L, Düking T, Schneider LV et al (2019) Ketogenic diet ameliorates axonal defects and promotes myelination in Pelizaeus-Merzbacher disease. Acta Neuropathol [internet]. Springer. Berlin Heidelberg 138:147-161 Available from: http://link.springer.com/10.1 007/s00401-019-01985-2

\section{Publisher's Note}

Springer Nature remains neutral with regard to jurisdictional claims in published maps and institutional affiliations.

Ready to submit your research? Choose BMC and benefit from:
- fast, convenient online submission
- thorough peer review by experienced researchers in your field
- rapid publication on acceptance
- support for research data, including large and complex data types
- gold Open Access which fosters wider collaboration and increased citations
- maximum visibility for your research: over 100M website views per year
At BMC, research is always in progress.
Learn more biomedcentral.com/submissions

